\title{
Permeabilidad de la barrera hematoencefálica al magnesio en preeclampsia
}

\author{
Cifuentes Rodrigo*; Paredes Ricardo*; Conde Agustín*; Romero Roberto**
}

RESUMEN OBJETIVO: Determinar si el magnesio cruza la barrera hematoencefálica en pacientes con preeclampsia.

MATERIAL Y METODOS: Se seleccionaron 41 embarazadas, 20 con preeclampsia (grupo de estudio) y 21 sin preeclampsia (grupo control). El grupo con preeclampsia recibió sulfato de magnesio en bolo inicial de 4 gr vía intravenosa y 1 gr/hora en dosis de mantenimiento. El grupo control no recibió sulfato de magnesio. A todas las pacientes se les practicó operación cesárea (por indicación obstétrica) y se les recolectó $1 \mathrm{~cm}^{3}$ de líquido cefalorraquídeo en el momento de la punción espinal para la anestesia regional.

Se mostraron además muestras de sangre periférica basal y 2 horas después. En todas las muestras se midió concentración de magnesio. Lo cual se hizo de manera ciega por uno de los investigadores.

RESULTADOS: Los niveles de magnesio en líquido cefalorraquídeo no fueron significativamente diferentes entre los dos grupos 4.88 $\mathrm{mEq} / \mathrm{L}$ (grupo control) y $5.58 \mathrm{mEq} / \mathrm{L}$ (grupo estudio) ( $\mathrm{p}=\mathbf{0 . 2 9}$ ). En contraste, los niveles séricos de magnesio fueron significativamente más altos en el grupo con preeclampsia $(5.91 \mathrm{mEq} / \mathrm{L})$ que en grupo control $(4.27 \mathrm{mEq} / \mathrm{l})(\mathrm{p}<0.003)$.

CONCLUSIONES: La barrera hematoencefálica parece no ser permeable al paso del ión magnesio en la preeclampsia.

PALABRAS CLAVES: Preeclampsia.

SUMMARY OBJECTIVE: To establish whether magnesium crosses the hematoencephalic barrier in patients with preeclampsia.

MATERIAL AND METHODS: 41 pregnant women were selected, from which 20 suffering from preeclampsia and 21 without preeclampsia (control group). The group with preeclampsia received magnesium sulfate in an initial $4 \mathrm{~g}$ large pills, intravenous and one gram/ hour in maintenance doses. The control group did not received sulphate of magnesium. All the patients had caesarean operation (by obstetric prescription) and $1 \mathrm{~cm}^{3}$ of cephalorachidian liquid was collected during the spinal puncture for local anesthesia.

Samples of basal peripheral blood were taken at that moment and two hours later. Blind measurements of Magnesium concentrations were carried out in all the samples.

RESULTS: Magnesium levels in cephalo-rachidian liquid were not significatively different in the two groups: $4,88 \mathrm{mEq} / \mathrm{L}$ (control group) and $5,58 \mathrm{mEq} / \mathrm{L}$ (group studied) $(\mathrm{p}=\mathbf{0 , 2 9})$. In contrast, magnesium sericeous levels were significatively higher in the group suffering from preeclampsia $(5,91 \mathrm{mEq} / \mathrm{L})$ than those of the control group $(4,27 \mathrm{mEq} / \mathrm{l})(\mathrm{p}<0,003)$.

CONCLUSIONS: Apparently the hemato-encephalic barrier is not permeable to the passage of the magnesium ion in the preeclampsia disorder.

KEY WORDS: Preeclampsia.

\section{Introducción}

El $\mathrm{MgSO}_{4}$ es la droga más usada en los Estados Unidos para la prevención o tratamiento de convulsiones en pacientes con preeclampsia y eclampsia. Existe una gran controversia acerca del mecanismo de acción del Sulfato de magnesio en el control de las convulsiones. Algunos (1-2) argumentan que el sulfato de magnesio tiene una acción anticonvulsivante periférica resultante de su habilidad para bloquear la transmisión neuromuscular por disminución de la liberación de acetilcolina

Servicio de Medicina Perinatal. Departamento de Ginecología y Obstetricia. Facultad de Salud. Universidad del Valle.

Jefe. Perinatal Research Branch. National Institute of Health. U.S.A. en respuesta a potenciales de acción nerviosa. Otros investigadores argumentan que los niveles de magnesio alcanzados durante el tratamiento de las convulsiones eclampticas no es tan alto como para causar parálisis muscular periférica y así explican la acción anticonvulsivante mediante un efecto central.

Estudios previos (3-4) han mostrado que el magnesio parecería cruzar la barrera hematoencefálica (HE), pero no se ha demostrado que un incremento en la concentración de magnesio en líquido cefalorraquídeo LCR provea un sitio de acción a nivel central.

El objetivo del presente estudio fue determinar si el magnesio cruza la barrera HE en cantidades significativas después de la terapia IV con $\mathrm{MgSO}_{4}$. 


\section{Pacientes y métodos}

Esta investigación fue realizada en el Hospital Universitario del Valle (Cali-Colombia), que es un Centro de Atención terciaria para la Región. El estudio fue aprobado por el Comité de Etica Médica de la Institución y todas las mujeres participantes dieron consentimiento por escrito.

El grupo de estudio consistió en 20 mujeres con diagnóstico de preeclampsia que fueron llevadas a operación cesárea mediante anestesia espinal y recibiendo sulfato de magnesio. Las mujeres fueron consideradas preeclampticas si las mediciones de la TA en 2 o más ocasiones que con al menos 6 horas de diferencia revelaron una tensión arterial sistólica (TAS) $\geqslant 140 \mathrm{mmHg}$ y una tensión arterial diastólica TAD $\geqslant 90 \mathrm{mmHg}$ después de la semana 20 de gestación, y proteinuria (proteínas > $0.3 \mathrm{gr}$ por litro en la orina). Todas ellas recibieron sulfato de magnesio en una dosis inicial de $4 \mathrm{gr}$ vía intravenosa y luego una dosis de mantenimiento de $1 \mathrm{gr} / \mathrm{hora}$. El grupo control consistió en 21 mujeres embarazadas normales sin complicaciones obstétricas y que no recibieron sulfato de magnesio y fueron llevadas a operación cesárea por distocia pélvica.

A todas las pacientes se les recolectó $1 \mathrm{cc}$. de líquido cefalorraquídeo en el momento de la punción espinal para anestesia regional de tipo espinal. Simultáneamente se recolectaron $10 \mathrm{cc}$. de sangre periférica venosa. Las muestras de LCR se centrifugaron por 10 minutos y posteriormente se congelaron a $-20^{\circ} \mathrm{C}$; las muestras de sangre se dejaron coagular para generar suero y después se centrifugaron por 10 minutos. Posteriormente fueron congeladas a $-20^{\circ} \mathrm{C}$.

Las concentraciones de Magnesio y el análisis de los datos fue realizado por uno de los investigadores blindado a los códigos del estudio.

El análisis comparativo de los datos fue realizado por el U-Mann-Whitney Test. Un valor de $P \leqslant 0.05$ fue considerado estadísticamente significativo.

\section{Resultados}

Las características demográficas maternas son mostradas en la tabla 1. El intervalo de tiempo transcurrido entre el inicio de la terapia con sulfato de magnesio y la operación cesárea en pacientes preeclampticas fue de 2 horas.

La tabla 2 presenta los niveles de magnesio en plasma y LCR en los grupos de estudio. El nivel sérico promedio de magnesio en el grupo recibió sulfato de magnesio y es significativamente más alto que los niveles séricos del grupo control $(\mathrm{P}<0.05)$. El nivel promedio en LCR de magnesio fue mayor en el grupo con preeclampsia comparado con el grupo control, pero no fue estadísticamente significativo; además hubo mucha sobreposición en los niveles de magnesio en LCR entre los dos grupos (Figura 1).

\section{Discusión y conclusiones}

En 1955 Pritchard (5) evaluó los niveles de magnesio en plasma y LCR de 10 pacientes preeclampticas-
Tabla 1

CARACTERISTICAS DEMOGRAFICAS MATERNAS AL INGRESO. NO HUBO DIFERENCIAS SIGNIFICATIVAS EN LOS DOS GRUPOS

\begin{tabular}{|lrc|}
\hline \multicolumn{1}{|c|}{$\begin{array}{c}\text { Preeclampsia } \\
(\mathbf{n = 2 0})\end{array}$} & $\begin{array}{c}\text { Controles } \\
(\mathbf{n = 2 1})\end{array}$ \\
\hline Edad (en años) & $22,8+5.5$ & $27+5.7$ \\
Paridad & $1.6+1.2$ & $2.1+0.9$ \\
Edad Gestacional & $35.3+3.7$ & $38.5+3.8$ \\
\hline
\end{tabular}

Tabla 2

NIVELES DE MAGNESIO EN SUERO Y EN LCR EN LAS DOS POBLACIONES ESTUDIADAS (PREECLAMPSIA Y CONTROL)

\begin{tabular}{|lccl|}
\hline & \multicolumn{1}{|c|}{$\begin{array}{r}\text { Preeclampsia } \\
\mathbf{N = 2 0}\end{array}$} & $\begin{array}{c}\text { Controles } \\
\mathbf{N = 2 1}\end{array}$ & \multicolumn{1}{c|}{$\mathbf{P}$} \\
\hline Suero $(\mathrm{MEg} / \mathrm{L})$ & $5.91+0.64$ & $4.27+0.36$ & $<0.05$ \\
LCR $(\mathrm{MEg} / \mathrm{L})$ & $\mathrm{LCR} 5.58+0.57$ & $4.88+0.31$ & $\mathrm{NS}$ \\
\hline
\end{tabular}

LCR = Líquido cefalorraquídeo

Valores son presentados como promedio \pm SE

eclampticas, tratadas con sulfato de magnesio intramuscular. Aunque él encontró que los niveles de magnesio en LCR fueron más altos en el grupo tratado con magnesio (2,65 Vs. $2,35 \mathrm{mEq} / \mathrm{L})$, no fue posible obtener un equilibrio entre el magnesio plasmático y LCR; además los niveles de magnesio en LCR no fueron dependientes de los niveles plasmáticos.

Thurnau y col. (3) evaluaron los niveles de magnesio en suero y LCR de 10 pacientes preeclampticas recibiendo sulfato de magnesio ( $6 \mathrm{gr}$ inicial y $2 \mathrm{gr} / \mathrm{h}$ ) y 11 controles; el tiempo promedio transcurrido entre el inicio del tratamiento con sulfato de magnesio y la operación cesárea fue 15.8 horas (rango: 4,5-72h). Los niveles promedio en suero y de magnesio en LCR promedio de magnesio fueron significativamente más altos en el grupo recibiendo sulfato de magnesio que el grupo no tratado.

Ellos concluyeron que "aunque solamente una pequeña cantidad de magnesio cruza la barrera HE después de al menos 4.5 horas de terapia con sulfato de magnesio este incremento fue estadísticamente significativo $(\mathrm{P}<0.001)$.

Recientemente, Moore y Col. (4), presentaron en el último Congreso Mundial de HTA en el embarazo, un reporte similar al de Thurnau. El grupo de estudio consistió de 10 mujeres con diagnóstico de hipertensión inducida por el embarazo (HIE), quienes recibían sulfato de magnesio (4 gr inicial y $1 \mathrm{gr} / \mathrm{h}$ ). El grupo control consistió en 10 mujeres sin PIH y que no recibieron sulfato de magnesio. Los niveles promedio de magnesio séricos y en LCR fueron significativamente más altos en grupo HIE que en el grupo control. No se mencionó el intervalo 
de tiempo transcurrido entre el inicio del tratamiento de sulfato de magnesio.

En nuestro trabajo, las muestras de LCR en las pacientes preeclampticas fueron tomadas 2 horas después de haberse iniciado la terapia con sulfato de magnesio. Estudios previos han mostrado que una dosis de 4 gr. de sulfato de magnesio causa una inmediata elevación de los niveles de magnesio $(1,6-2,1 \mathrm{mEq} / \mathrm{L}$ a $7-9 \mathrm{mEq} / \mathrm{L})$ séricos. Transferencia intracelular del ion y la eliminación por el riñón causa una caída en la concentración plasmática a 4-5 mEq/L 1 hora después de la inyección. Potencialmente la dosis de mantenimiento de $1 \mathrm{gr} /$ hora mantiene los niveles séricos de sulfato de magnesio estables; el hecho que en nuestro estudio los niveles de magnesio en LCR no fueran estadísticamente significativo entre los dos grupos sugeriría que inicialmente ( 2 horas) el magnesio no pasaría la barrera H-E y su acción anticonvulsivante se debería exclusivamente a una acción periférica. Los estudios previos demostraron que el sulfato de magnesio atravesaría la barrera HE pero varias horas después (al menos 4,5 horas) de iniciada la terapia. Sin embargo, aceptando este hecho, persistiría el interrogante, si un nivel incrementado de magnesio en LCR refleja un sitio de acción a nivel central (sistema nervioso). La polémica continuará.

En conclusión, nuestro estudio sugiere que el magnesio no atraviesa la barrera HE en las dos horas siguientes al inicio de la terapia con sulfato de magnesio, a pesar de tener "niveles séricos terapéuticos".

Obsérvese que no hay diferencia en los niveles de magnesio LCR en las dos poblaciones estudiadas. En cambio, los niveles séricos de Mg sí son estadísticamente mayores en el grupo con preeclampsia.

\section{BIBLIOGRAFIA}

1. Donaldson JO. Does magnesium sulfate treat eclamptic convulsions? Clin Neuropharmacol 1986; 9: 37-45.

2. Kaplan PW., Lesser RP., Fisher RS et al. No magnesium sulfate should not be used in treating eclamptic convulsions. Arch. Neurol. 1988; 45: 1361-1364.

3. Thurnau GR., Kemp DB., Jarris A. Cerebropinal fluid levels of magnesium in patients with preeclampsia after treatment with intravenous magnesium sulfate: A preliminary report. Am. Obstet. Gynecol. 1987; 157: 1435-1438.

4. Moore P., Belfort MA., Kirshan B. Cerebrospinal fluid magnesium levels in patients reciving magnesium sulfate for pregnancy induced hypertension. XIII Congreso Mundial de Hipertensión y Embarazo. Buenos Aires. Argentina, Nov. 30, 1992.

5. Pritchard JA. The use of the magnesium ion in the management of eclamtogenic toxemias. Surg. Gynecol. Obstet. 1955; 100: 131. 